\title{
Structural-parametric model of electromagnetoelastic actuator for nanomedicine and nanobiotechnology
}

\begin{abstract}
The generalized parametric structural schematic diagram, the generalized structuralparametric model, the solution of wave equation, the generalized matrix transfer function of the electromagnetoelastic actuator are determined. The dynamic and static characteristics of the actuator are obtained. The parametric structural schematic diagram and the transfer functions are determined for calculation of the control systems of the nanomedicine and the nanobiotechnology. The structural-parametric models of the piezoactuator for the transverse, longitudinal, shift piezoelectric effects are constructed.
\end{abstract}

Keywords: structural-parametric model, electromagnetoelastic actuator, piezoactuator, parametric structural schematic diagram, deformation
Volume 3 Issue 4 - 2017

\begin{abstract}
Afonin SM
Department of Intellectual Technical Systems, National Research University of Electronic Technology (MIET), Russia

Correspondence: Afonin SM, Department of Intellectual Technical Systems, National Research University of Electronic Technology (MIET), Moscow, Russia, Email learner0I@mail.ru
\end{abstract}

Received: October 27, 2017 | Published: November 16, 2017

\section{Introduction}

The electromagnetoelastic actuator of the piezoeffect, the piezomagnetic effect, the electrostriction or the magnetostriction effect is used for precise alignment in the nanomedicine, the nanobiotechnology and the adaptive optics. ${ }^{1-32}$ The piezoactuator on the inverse piezoeffect is serves for the actuation of mechanisms or the management, converts electrical signals into displacement and force. ${ }^{1-8}$ The piezoactuator for the nanomechanics is provided the displacement from nanometers to tens of micrometers, a force to $1000 \mathrm{~N}$. The piezoactuator is used for research in the nanomedicine and the nanobiotechnology for the scanning tunneling microscopes, scanning force microscopes and atomic force microscopes. ${ }^{14-32}$ In the present paper the generalized structural-parametric model and the generalized parametric structural schematic diagram of the electromagnetoelastic actuator are constructed by solving the wave equation with the Laplace transform for the equation of the electromagnetolasticity, the boundary conditions on loaded working surfaces of the actuator, the strains along the coordinate axes. The transfer functions and the parametric structural schematic diagrams of the piezoactuator are obtained from the generalized structural-parametric model. $\operatorname{In}^{6,7}$ was determined the solution of the wave equation of the piezoactuator. In the ${ }^{14-16,30,31}$ were obtained the structural-parametric models, the schematic diagrams for simple piezoactuator and were transformed to the structural-parametric model of the electromagnetoelastic actuator. The structural-parametric model of the electroelastic actuator was determined in contrast electrical equivalent circuit for calculation of piezoelectric transmitter and receiver. ${ }^{9-12} \operatorname{In}^{8,27}$ was used the transfer functions of the piezoactuator for the decision problem absolute stability conditions for a system controlling the deformation of the electromagnetoelastic actuator. The elastic compliances and the mechanical and adjusting characteristics of the piezoactuator were found in ${ }^{18,21-23,28,29}$ for calculation its transfer functions and the structural-parametric models. The structural-parametric model of the multilayer and compound piezoactuator was determined in ${ }^{18-20}$ In this paper is solving the problem of building the generalized structural parametric model and the generalized parametric structural schematic diagram of the electromagnetoelastic actuator for the equation of electromagnetoelasticity.

\section{Structural-parametric model of electromagnetoelastic actuator}

The general structural-parametric model and the parametric structural schematic diagram of the electromagnetoelastic actuator are obtained. In the electroelastic actuator are presented six stress components $T_{1}, T_{2}, T_{3}, T_{4}, T_{5}, T_{6}$, the components $T_{1}$ $T_{3}$ re related to extension-compression stresses, $T_{4}-T_{6}$ to shear stresses. For the electroelastic actuator its deformation corresponds to stressed state. For polarized piezoceramics PZT the matrix state equations ${ }^{12,14}$ connected the electric and elastic variables have the form two equations, then the first equation describes the direct piezoelectric effect, the second - the inverse piezoelectric effect

$$
\begin{aligned}
& \mathbf{S}=\mathbf{s}^{E} \mathbf{T}+\mathbf{d}^{t} \mathbf{E} \\
& \mathbf{S}=\mathbf{s}^{E} \mathbf{T}+\mathbf{d}^{t} \mathbf{E}
\end{aligned}
$$

Where $\mathbf{D}$ is the column matrix of electric induction; $\mathbf{S}$ is the column matrix of relative deformations; $\mathbf{T}$ is the column matrix of mechanical stresses; $\mathbf{E}$ is the column matrix of electric field strength; $\mathbf{s}^{E}$ is the elastic compliance matrix for $E=$ const ; $\stackrel{a}{T}^{T}$ is the matrix of dielectric constants for $T=$ const ; $\mathbf{d}^{t}$ is the transposed matrix of the piezoelectric modules. The piezoactuator (piezoplate) has the following properties: $\delta$ - the thickness, $h$-the height, $b$-the width, respectively $l=\{\delta, h, b$ the length of the piezoactuator for the longitudinal, transverse and shift piezoeffect. The direction of the polarization axis $P$, i.e., the direction along which polarization was performed, is usually taken as the direction of axis 3 . The equation of the inverse piezoeffect for controlling voltage $\mathrm{e}^{6,12}$ has the form

$$
\mathrm{Si}=\mathrm{dmi} \Psi \mathrm{m}(t)+\mathrm{s}_{i j}{ }_{i j} \mathrm{Tj}(x, t)
$$

$S i=\partial \xi(x, t) / \partial x, \Psi m(t)=E m(t)=U(t) / \delta$

Where $S_{i}$ is the relative displacement of the cross section of the piezoactuator along axis $i, \Psi_{m}(t)$ is the control parameter along axis $m, d_{m i}$ is the piezomodule, $E_{m}(t)$ is the electric field strength along axis $m, U(t)$ is the voltage between the electrodes of actuator, $s_{i j}^{\Psi}$ is the elastic compliance, $T_{j}$ is the mechanical stress along axis 
$j$ and $i, j=1,2, \ldots, 6 ; m=1,2,3$. The main size $l=\{\delta, h, b$ for the piezoactuator, respectively, the thickness, the height, the width for the longitudinal, transverse, shift piezoeffect. For calculation of actuator is used the wave equation ${ }^{6,7,12,14}$ for the wave propagation in a long line with damping but without distortions. After Laplace transform is obtained the linear ordinary second-order differential equation with the parameter $p$, whereupon the original problem for the partial differential hyperbolic equation of type using the Laplace transform is reduced to the simpler problem ${ }^{6,13}$ for the linear ordinary differential equation

$$
\frac{d^{2} \Xi(x, p)}{d x^{2}}-\gamma^{2} \Xi(x, p)=0
$$

With its solution

$$
\Xi(x, p)=C e^{-x \gamma}+B e^{x \gamma}
$$

Where $\Xi(x, p)$ is the Laplace transform of the displacement of the section of the piezoactuator, $\gamma=p / c^{\Psi}+\alpha$ is the propagation coefficient, $c^{\Psi}$ is the sound speed for $\Psi=$ const , $\alpha$ is the damping coefficient, $\Psi$ is the control parameter: $E$ for the voltage control, $D$ for the current control, $H$ for the magnet field strength control From $(3,5)$, the boundary conditions on loaded surfaces, the strains along the axes the system of equations for the generalized structuralparametric model and the generalized parametric structural schematic diagram Figure 1 of the actuator are determined

$$
\Xi_{2}(p)=\left(\frac{1}{M_{2} p^{2}}\right)\left\{-F_{2}(p)+\left(\frac{1}{\chi_{i j}^{\Psi}}\right)\left[\left(\frac{\gamma}{d_{m i} \Psi_{m}(p)-}-\left(\frac{\gamma}{\operatorname{sh}(l \gamma)}\right)\left[\operatorname{ch}(l \gamma) \Xi_{2}(p)-\Xi_{1}(p)\right]\right]\right\}\right.
$$

$$
\begin{gathered}
\text { where } \quad \chi_{i j}^{\Psi}=\frac{s_{i j}^{\Psi}}{S_{0}} d_{m i}=\left\{\begin{array}{l}
d_{33}, d_{31}, d_{15} \\
g_{33}, g_{31}, g_{15}, \\
d_{33}, d_{31}, d_{15}
\end{array} \quad \Psi_{m}=\left\{\begin{array}{l}
E_{3}, E_{3}, E_{1} \\
D_{3}, D_{3}, D_{1} \\
H_{3}, H_{3}, H_{1}
\end{array}\right.\right. \\
s_{i j}^{\Psi}=\left\{\begin{array}{l}
s_{33}^{E}, s_{11}^{E}, s_{55}^{E} \\
s_{33}^{D}, s_{11}^{D}, s_{55}^{D}, \\
s_{33}^{H}, s_{11}^{H}, s_{55}^{H}
\end{array} \quad l=\left\{\delta, h, b, \quad c^{\Psi}=\left\{c^{E}, c^{D}, c^{H},\right.\right.\right.
\end{gathered}
$$

$\gamma^{\Psi}=\left\{\gamma^{E}, \gamma^{D}, \gamma^{H}, d_{m i}\right.$ isthecoefficientoftheelectromagnetolasticity (piezomodule or coefficient of magnetostriction). Figure 1 shows the generalized parametric structural schematic diagram of the electromagnetoelastic actuator corresponding to the set of equations (6). The generalized transfer functions of the electromagnetoelastic actuator are the ratio of the Laplace transform of the displacement of the face actuator and the Laplace transform of the corresponding control parameter or the force at zero initial conditions. From (6) the generalized matrix equation of the transfer functions of electromagnetolastic actuator

$$
\left(\begin{array}{l}
\Xi_{1}(p) \\
\Xi_{2}(p)
\end{array}\right)=\left(\begin{array}{lll}
W_{11}(p) & W_{12}(p) & W_{13}(p) \\
W_{21}(p) & W_{22}(p) & W_{23}(p)
\end{array}\right)\left(\begin{array}{c}
\Psi_{m}(p) \\
F_{1}(p) \\
F_{2}(p)
\end{array}\right)
$$

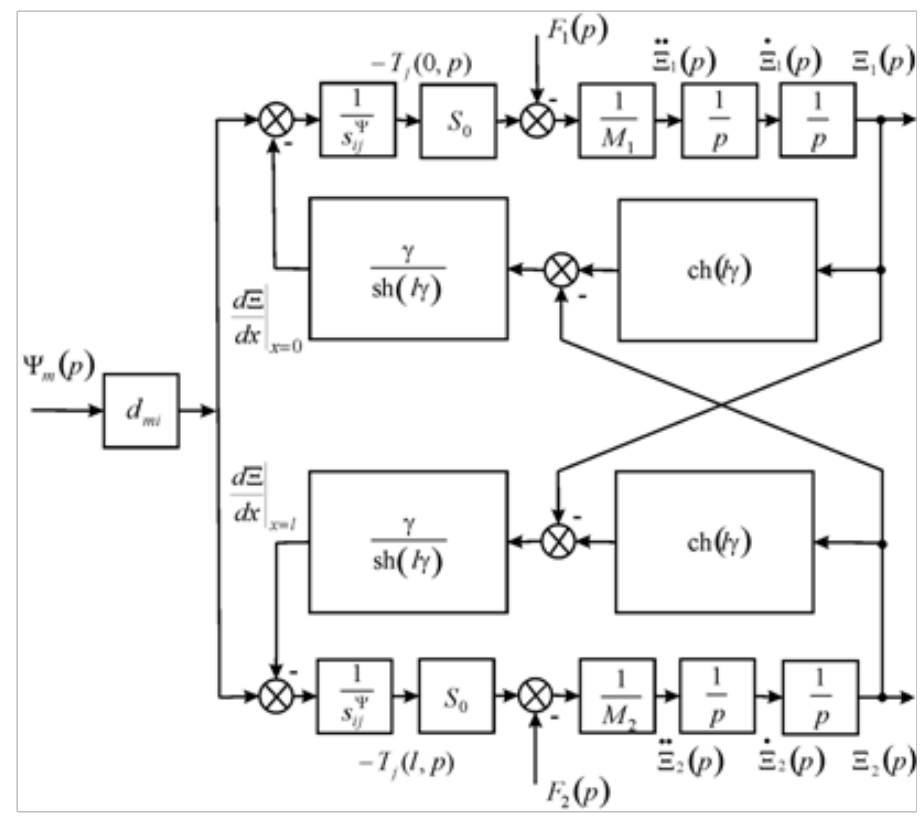

Figure I Generalized parametric structural schematic diagram of the electromagnetoelastic actuator.

For $m \ll M_{1}$ and $m \ll M_{2}$ the static displacement of the faces of the piezoactuator for the transverse piezoeffect are obtained

$$
\begin{aligned}
& \xi_{1}(\infty)=\lim _{\substack{p \rightarrow 0 \\
\alpha \rightarrow 0}} \frac{p W_{11}(p) U_{0}}{\delta p}=\frac{d_{31} h U_{0} M_{2}}{\delta\left(M_{1}+M_{2}\right)} \\
& \hat{\imath}_{2}(\infty)=\lim _{p \rightarrow 0} \frac{p W_{21}(p) U_{0}}{\ddot{a} p}=\frac{d_{31} h U_{0} M_{1}}{\ddot{a}\left(M_{1}+M_{2}\right)}
\end{aligned}
$$

For the piezoactuator from PZT under the transverse piezoeffect at $m<M_{1}$ and $m<<M_{2}, \quad d_{31}=2.5 \cdot 10^{-10} \mathrm{~m} / \mathrm{V}, \quad h / \delta=20$ , $U=120 \mathrm{~V}, M_{1}=2 \mathrm{~kg}$ and $M_{2}=8 \mathrm{~kg}$ the static displacement of the faces are determined $\hat{\mathrm{i}}_{1}(\infty)=480 \mathrm{~nm}, \hat{\mathrm{i}}_{2}(\infty)=120 \mathrm{~nm}$, $\hat{\mathrm{i}}_{1}(\infty)+\hat{\mathrm{i}}_{2}(\infty)=600 \mathrm{~nm}$. For the approximation of the hyperbolic cotangent by two terms of the power series in transfer function (7) the following expressions of the transfer function of the piezoactuator is obtained for the elastic-inertial load at $M_{1} \rightarrow \infty, m<<M_{2}$ under the transverse piezoeffect

$$
\begin{aligned}
& W(p)=\frac{\Xi_{2}(p)}{U(p)}=\frac{d_{31} h / \delta}{\left(1+C_{e} / C_{11}^{E}\right)\left(T_{t}^{2} p^{2}+2 T_{t} \xi_{t} p+1\right)} \\
& T_{t}=\sqrt{M_{2} /\left(C_{e}+C_{11}^{E}\right)}, \xi_{t}=\alpha h^{2} C_{11}^{E} /\left(3 c^{E} \sqrt{M\left(C_{e}+C_{11}^{E}\right)}\right)
\end{aligned}
$$

Where $U(p)$ is the Laplace transform of the voltage, $T_{t}$ is the time constant and $\xi_{t}$ is the damping coefficient of the piezoactuator. The expression for the transient response of the voltage-controlled piezoactuator for the elastic-inertial load under the transverse piezoeffect is determined 


$$
\begin{aligned}
& \xi(t)=\xi_{m}\left[1-\frac{e^{-\frac{\xi_{t} t}{T_{t}}}}{\sqrt{1-\xi_{t}^{2}}} \sin \left(\omega_{t} t+\phi_{t}\right)\right] \\
& \hat{\mathbf{1}}_{m}=\frac{d_{31}(h / \ddot{\mathrm{a}}) U_{m}}{1+C_{e} / C_{11}^{E}}, \omega_{t}=\frac{\sqrt{1-\xi_{t}^{2}}}{T_{t}}, \phi_{t}=\operatorname{arctg}\left(\frac{\sqrt{1-\xi_{t}^{2}}}{\xi_{t}}\right)
\end{aligned}
$$

Where $\xi_{m}$ is the steady-state value of displacement of the piezoactuator, $U_{m}$ is the amplitude of the voltage. For the voltagecontrolled piezoactuator from the piezoceramics PZT under the transverse piezoelectric effect for the elastic-inertial load $M_{1} \rightarrow \infty$ , $m<<M_{2}$ and input voltage with amplitude $U_{m}=100 \mathrm{~V}$ at $d=2.5 \cdot 10^{-10} \mathrm{~m} / \mathrm{V}, \quad h / \delta=20, \quad M_{2}=9 \mathrm{~kg}, \quad C_{11}^{E}=2 \cdot 10^{7} \mathrm{~N} / \mathrm{m}$, 31

$C_{e}=0.5 \cdot 10^{7} \mathrm{H} / \mathrm{m}$ are obtained values $\hat{1}_{m}=400 \mathrm{~nm}, T_{t}=0.6 \cdot 10^{-3}$ c

\section{Results \& discussion}

The structural-parametric model and parametric structural schematic diagrams of the voltage-controlled piezoactuator for the longitudinal, transverse and shift piezoelectric effects are determined from the generalized structural-parametric model of the electromagnetoelastic actuator for the nanomedicine and the nanobiotechnology with the replacement of the following parameters

$$
\Psi_{m}=\left\{E_{3}, E_{3}, E_{1}, \quad d_{m i}=\left\{d_{33}, d_{31}, d_{15}, \quad s_{i j}^{\Psi}=\left\{s_{33}^{E}, s_{11}^{E}, s_{55}^{E},\right.\right.\right.
$$

$l=\{\ddot{a}, h, b$

The generalized structural-parametric model, the generalized parametric structural schematic diagram and the matrix equation of the electromagnetoelastic actuator are obtained from the solutions of the wave equation with the Laplace transform and from its deformations along the coordinate axes. From the generalized matrix equation for the transfer functions of the electromagnetoelastic actuator after algebraic transformations are constructed the matrix equations of the piezoactuator for the longitudinal, transverse and shift piezoelectric effects.

\section{Conclusion}

The generalized structural-parametric model, the generalized parametric structural schematic diagram, the matrix equation of the electromagneto elastic actuator for the nanomedicine and the nanobiotechnology are obtained. The structural-parametric model, the matrix equation and the parametric structural schematic diagram of the piezoactuator for the transverse, longitudinal, shift piezoelectric effects are obtained from the generalized structural-parametric model of the electromagneto elastic actuator. From the solution of the wave equation, from the equation of the electromagnetolasticity and the deformations along the coordinate axes the generalized structuralparametric model and the generalized parametric structural schematic diagram of the electromagnetoelastic actuator are constructed for the control systems for the nanomedicine and the nanobiotechnology. The deformations of the actuator are described by the matrix equation for the transfer functions of the actuator.

\section{Acknowledgements}

None.

\section{Conflict of interest}

The author declares no conflict of interest.

\section{References}

1. Schultz J, Ueda J, Asada H. Cellular Actuators. England: ButterworthHeinemann Publisher; 2017. 382 p.

2. Uchino K. Piezoelectric actuator and ultrasonic motors. USA: Kluwer Academic Publisher; 1997;1:350.

3. Przybylski J. Static and dynamic analysis of a flextensional transducer with an axial piezoelectric actuation. Engineering structures. 2015;84:140-151.

4. Ueda J, Secord T, Asada HH. Large effective-strain piezoelectric actuators using nested cellular architecture with exponential strain amplification mechanisms. IEEE/ASME Transactions on Mechatronics. 2010;15(5):770-782.

5. Karpelson M, Wei GY, Wood RJ. Driving high voltage piezoelectric actuators in microrobotic applications. Sensors and Actuators A: Physical. 2012;176:78-89.

6. Afonin SM. Solution of the wave equation for the control of an elecromagnetoelastic transduser. Doklady mathematics. 2006;73(2):307313

7. Afonin SM. Structural parametric model of a piezoelectric nanodisplacement transduser. Doklady physics. 2008;53(3):137-143.

8. Afonin SM. Stability of strain control systems of nano-and microdisplacement piezotransducers. Mechanics of solids. 2014;49(2):196-207.

9. Talakokula V, Bhalla S, Ball RJ, et al. Diagnosis of carbonation induced corrosion initiation and progressionin reinforced concrete structures using piezo-impedance transducers. Sensors and Actuators A: Physical. 2016;242:79-91.

10. Yang Y, Tan L. Equivalent circuit modeling of piezoelectric energy harvesters. Journal of intelligent material systems and structures. 2009;20(18):2223-2235.

11. Cady WG. Piezoelectricity: An introduction to the theory and applications of electromechancial phenomena in crystals. 1st ed. USA: McGraw-Hill Book Company; 1946. 806 p.

12. Physical Acoustics: Principles and Methods. In: Mason W editor. USA: Academic Press; 1946. 532 p

13. Zwillinger D. Handbook of Differential Equations. 1st ed. USA: Academic Press; 1989. 801 p.

14. Afonin SM. Structural-parametric model and transfer functions of electroelastic actuator for nano- and microdisplacement. In: Parinov editor. USA: Nova Science; 2015. p. 225-242.

15. Afonin SM. Structural-parametric model electromagnetoelastic actuator nano- and microdisplacement for precision engineering. Precision Engineering and Technology. 2016;3(6):110-119.

16. Afonin SM. Structural-parametric models and transfer functions of electromagnetoelastic actuators nano- and microdisplacement for mechatronic systems. International Journal of Theoretical and Applied Mathematics. 2016;2(2):52-59.

17. Afonin SM. Parametric structural diagram of a piezoelectric converter Mechanics of solids. 2002;37(6):85-91. 
18. Afonin SM. Deformation, fracture, and mechanical characteristics of compound piezoelectric transducer. Mechanics of solids. 2003;38(6):7882.

19. Afonin SM. Parametric block diagram and transfer functions of a composite piezoelectric transducer. Mechanics of solids. 2004;39(4):119 127.

20. Afonin SM. Generalized parametric structural model of a compound elecromagnetoelastic transduser. Doklady physics. 2005;50(2):77-82.

21. Afonin SM. Design static and dynamic characteristics of a piezoelectric nanomicrotransducers. Mechanics of solids. 2010;45(1):123-132.

22. Afonin SM. Electromechanical deformation and transformation of the energy of a nano-scale piezomotor. Russian engineering research. 2011;31(7):638-642.

23. Afonin SM. Electroelasticity problems for multilayer nano- and micromotors. Russian engineering research. 2011;31:842.

24. Afonin SM. Nano- and micro-scale piezomotors. Russian engineering research. 2012;32(7-8):519-522.

25. Afonin SM. Optimal control of a multilayer submicromanipulator with a longitudinal piezo effect. Russian engineering research. 2015;35(12):907-910.
26. Afonin SM. Block diagrams of a multilayer piezoelectric motor for nanoand microdisplacements based on the transverse piezoeffect. Journal of computer and systems sciences international. 2015;54(3):424-439.

27. Afonin SM. Absolute stability conditions for a system controlling the deformation of an elecromagnetoelastic transduser. Doklady mathematics. 2006;74(3):943-948.

28. Afonin SM. Elastic compliances and mechanical and adjusting characteristics of composite piezoelectric transducers. Mechanics of solids. 2007;42(1):43-49.

29. Afonin SM. Static and dynamic characteristics of a multy-layer electroelastic solid. Mechanics of solids. 2009;44(6):935-950.

30. Afonin SM. Structural-parametric model electromagnetoelastic actuator nanodisplacement for mechatronics. International Journal of Physics. 2017;5(1):9-15.

31. Afonin SM. A structural-parametric model of electroelastic actuator for nano- and microdisplacement of mechatronic system. In: Bartul Z, et al. editors.USA: Nova Science; 2017. p. 259-284.

32. Springer Handbook of Nanotechnology. In: Bhushan B editor. USA: Springer; 2004. $1222 \mathrm{p}$ 